\title{
Tracking the Fluorescence Lifetimes of Cesium Lead Halide Perovskite Nanocrystals During their Synthesis Using a Fully Automated Optofluidic Platform
}

\author{
Ioannis Lignos, ${ }^{\text {a }, c^{*}}$ Richard M. Maceiczyk, ${ }^{a}$ Maksym V. Kovalenko, ${ }^{\text {bd }}$ and Stavros Stavrakis ${ }^{{ }^{*}}$ \\ a Institute for Chemical and Bioengineering, Department of Chemistry and Applied Biosciences, ETH \\ Zurich, Vladimir-Prelog-Weg 1, 8093, Switzerland; \\ ${ }^{b}$ Laboratory of Inorganic Chemistry, Department of Chemistry and Applied Biosciences, ETH Zurich, \\ Vladimir-Prelog-Weg 1, 8093, Switzerland. \\ ${ }^{c}$ Present address: Department of Chemical Engineering, Massachusetts Institute of Technology, 77 \\ Massachusetts Avenue, Cambridge, MA 02139, U.S.A \\ ${ }^{\mathrm{d}}$ Empa - Swiss Federal Laboratories for Materials Science and Technology, Ueberlandstrass 129, CH- \\ 8600, Duebendorf, Switzerland
}

*Corresponding authors: ilignos@mit.edu, stavros.stavrakis@chem.ethz.ch

This document is the accepted manuscript version of the following article: Lignos, I., Maceiczyk, R. M., Kovalenko, M. V., \& Stavrakis, S. (2019). Tracking the fluorescence lifetimes of cesium lead halide perovskite nanocrystals during their synthesis using a fully automated optofluidic platform. Chemistry of Materials. https://doi .org/10.1021/acs. chemmater.9b03438 
Abstract

Semiconductive metal halide perovskites have opened exciting opportunities in a range of optoelectronic applications including solar cells, photodetectors, lasers and light emitting devices. Recently, all-inorganic cesium lead halide $\left(\mathrm{CsPbX}_{3} ; \mathrm{X}=\mathrm{Cl}, \mathrm{Br}, \mathrm{I}\right)$ nanocrystals have become attractive light sources due to their high photoluminescence quantum yields, narrow emission linewidths and emission color tunable over the entire visible region. Their radiative rates are higher (i.e. luminescence lifetimes shorter) than those of more conventional quantum dots, making perovskite NCs brighter emitters, highly attractive as both classical and quantum light sources. Multiple factors - primarily synthesis parameters and post-synthetic experience govern the observed radiative lifetime and other optical characteristics. High-throughput experimentation in microfluidic platforms equipped with in-line optical characterization had proven to be highly instrumental for rapid and accurate assessment of optical properties, mainly in a steady-state mode. Thus far, in-line measurement of the radiative lifetimes and hence the proper use of high-trhoughput experimentation for tailored engineering of radiative rates had been elusive. Herein, we showcase a fully automated optofluidic platform that integrates time-correlated single photon counting measurements in droplet-based flow for the rigorous extraction of fluorescence lifetimes of $\mathrm{CsPbX}_{3}$ nanocrystals. Sensitivites of the experimental setup allow for measurements at a single-droplet level. Such concurrent time-resolved photoluminescence allow mapping the parametric space in time-efficient manner ( $\sim 1000$ lifetime measurements in 5 hours of operation) and with high reagent economy ( $200 \mathrm{~nL}$ reaction volume per measurement). We elucidated the effects of composition and ratios of judiciously chosen reagents, as well as temperature on the fluorescence lifetimes $(5-42 \mathrm{~ns})$. Specifically, the average lifetime as well as the emission spectra of all halide compositions tested was strongly dependent on $\mathrm{Pb}$-to-Cs variations. Accordingly a correlation between the steady-state luminescence amplitude and fluorescence lifetimes was established, thus providing a simple method to differentiate between the photoluminescence quantum yields, concentration effects and effects due to the non-radiative recombination at the surface traps. Such microfluidic tool will aid in the physicochemical and photophysical properties of diverse perovskite nanocrystals and other luminescent materials produced in the liquid-state synthesis. 


\section{Introduction}

Fluorescence spectroscopy has been paramount as a routine characterization tool in research laboratories focusing on the synthesis and applications of semiconductor nanocrystals (NCs), also known as quantum dots (QDs). ${ }^{1-2}$ QDs have been studied intensely due to their unique electronic and optical properties and potential utility in biological luminescent labeling, ${ }^{3-4}$ electrically and optically driven light emitting devices, ${ }^{4-12}$ photodetectors ${ }^{13-16}$ and photovoltaic devices. ${ }^{17-23}$ It is well recognized that reaction parameters during the bottom-up synthesis of QDs (concentrations and molar ratios of precursors and ligands, temperature $v s$. reaction time profile) have a strong influence on the size and morphology of the QDs and the state of surface, hence drastically altering the photoluminescence quantum yield (PL QY) and other characteristics of the formed particles. ${ }^{23}$ In general, measurement of the decay in PL of species after excitation by a short optical pulse allows for the determination of both radiative $\left(\mathrm{k}_{\mathrm{r}}\right)$ and non-radiative rate $\left(\mathrm{k}_{\mathrm{nr}}\right)$ coefficients, which control and define the PL quantum efficiency. ${ }^{24}$ Accordingly, the ability to perform on-the-fly time-correlated single photon counting (TCSPC) measurements, when synthesizing nanoparticles, will significantly enhance useful information content by providing an absolute measure independent of sample concentration. For example, evaluation of PL lifetime components will reveal whether an increase in the time-integrated emission intensity is due to an increase in PL QY (Eq. 1) or the product concentration, with the latter not causing any change in the average fluorescence lifetime $(\tau)(\mathrm{Eq}$. 2). ${ }^{24}$

$$
\begin{aligned}
& P L Q Y=\frac{k_{r}}{k_{r}+k_{n r}} \\
& \tau=\frac{1}{k_{r}+k_{n r}}
\end{aligned}
$$

Lead halide perovskite NCs (LHP NCs) are exceptional materials for a range of nanomaterial-based optoelectronic applications, including photonic sources ${ }^{2,10,25-33}$ and light harvesters. ${ }^{19-21,34-35}$ Numerous synthetic approaches have been reported for the formation of colloidal $\mathrm{APbX}_{3} \mathrm{NCs}$ with $\mathrm{Cs}$, formamidinium (FA) and methylammonium (MA) or mixtures thereof as ions of the A-site and various halides. ${ }^{2,31,34,36-45}$ In recent years, all-inorganic $\mathrm{CsPbX}_{3} \mathrm{NCs}$ have attracted enormous attention due to their near-unity QYs and facile compositional tuning of their emission and absorption energies across the entire visible region of the electromagnetic spectrum. ${ }^{2}$ Several methodologies have been reported for the elucidation of their nucleation and growth mechanisms, ${ }^{46-47}$ surface chemistry ${ }^{48-53}$ and photophysics. ${ }^{54-60}$ However, a detailed understanding of the reaction parameters that govern their physicochemical and photophysical characteristics is limited by the rapid kinetics of nucleation and growth associated with ionic-metathesis- 
based processes. ${ }^{2,46}$ Additionally, the phase instability of the $3 \mathrm{D}$ polymorph of $\mathrm{CsPbI}_{3}$ or $\mathrm{CsPb}(\mathrm{Br} / \mathrm{I})_{3} \mathrm{NCs}$ limits photophysical characterization for certain cationic or halide compositions. ${ }^{61}$

Near-future anticipated applications of LHP NCs are in liquid crystal displays (LCD) ${ }^{62}$ exploiting bright narrow-band PL or in light emitting diode (LED) displays ${ }^{63}$ making use of electroluminescence. Beyond these classical applications, information regarding the fluorescence lifetimes of perovskite NCs is significant for the development of quantum light sources, such as single-photon LEDs ${ }^{64}$ or for quantum information technologies. ${ }^{60,65} \mathrm{CsPbX}_{3} \mathrm{NCs}$ have short radiative lifetimes (few ns at room temperature), typically an order of magnitude faster than InP or CdSe QDs and three orders of magnitude faster at cryogenic temperatures, which makes them ideal candidates for the development of single-photon sources. ${ }^{56}$, ${ }^{66}$ Perovskite NCs are thus far the most optically coherent chemically synthesized single-photon sources. ${ }^{60}$ An important aspect is that the profile of the PL decay and the average radiative lifetime can be related to NC stability. A recent study by Bodnarchuk et al..$^{43}$ exemplifies the effects of surface passivation and aging on PL lifetimes and PL QYs, elucidated by standard ex-situ optical measurements. It is therefore a highly desired prerequisite to develop a strategy for the direct in-situ and cost-efficient evaluation of the PL lifetimes in response to the synthesis conditions, isolation and purification steps.

As noted, TCSPC measurements can provide a detailed analysis of excited state dynamics through the evaluation of PL decay time components. ${ }^{24}$ That said, NC characterization is normally performed in an offline manner (for NCs synthesized in flask reactors), using methods based on time-integrated and timeresolved fluorescence, absorption spectroscopy and light scattering. ${ }^{67-68}$ Automation of batch synthetic processes, together with the rapid optimization of the physicochemical and photophysical properties of the resulting NCs is almost always challenging, due to the need for extensive sample preparation, the difficulties associated with detector integration and the need for particle purification post-synthesis. ${ }^{68-69}$ This is particularly crucial for the discovery and development of more complex perovskite compositions, such as emerging double perovskite $\mathrm{NCs}$, with recent examples being $\mathrm{Cs}_{2} \mathrm{Ag}_{1-\mathrm{x}} \mathrm{Na}_{\mathrm{x}} \mathrm{InCl}_{6}{ }^{70-71}, \mathrm{Cs}_{2} \mathrm{AgIn}_{\mathrm{x}} \mathrm{Bi}_{1-\mathrm{x}} \mathrm{Cl}_{6}{ }^{72}$ and $\mathrm{Cs}_{2} \mathrm{AgBiX}_{6}{ }^{73} \mathrm{In}$ such systems, PL QY and PL lifetimes are deternimed by a complex interplay between doping strategies, B-site ions and reaction conditions, which makes the thorough parametric screening prohibitively slow with conventional flask synthesis and post-synthetic optical characterization. Conversely, microfluidic platforms incorporating optical detection systems have been shown to allow for the controlled synthesis of a wide range of NCs, together with efficient and real-time characterization of material properties. ${ }^{31,44-46,74-86}$ In this regard, most efforts have focused on in-line implementations of techniques such as fluorescence and UV-Visible absorption, ${ }^{75,87}$ X-ray scattering ${ }^{88}$ and correlation spectroscopies, ${ }^{89}$ with information regarding particle size, shape and size distribution being accessible.

Recently, we reported the microfluidic syntheses of $\mathrm{CsPbX}_{3}, \mathrm{FAPbX}_{3}$ and $\mathrm{Cs}_{\mathrm{x}} \mathrm{FA}_{1-\mathrm{x}} \mathrm{Pb}\left(\mathrm{Br}_{1-\mathrm{y}} \mathrm{I}_{\mathrm{y}}\right)_{3} \mathrm{NCs}$, together with rigorous and ultra-fast mapping of reaction parameter space, including molar ratios of cation 


\section{Experimental}

\section{Materials}

Cesium carbonate $\left(\mathrm{Cs}_{2} \mathrm{CO}_{3}\right.$, Aldrich, $\left.99.9 \%\right)$, lead bromide $\left(\mathrm{PbBr}_{2}, \mathrm{ABCR}, 98 \%\right)$, lead iodide $\left(\mathrm{PbI}_{2}, \mathrm{ABCR}\right.$, 99.999\%), 1-octadecene (ODE, 90\%), oleic acid (OA, Sigma Aldrich, 90\%), oleylamine (OLA, Acros, $\geq$ 96\%), perfluorooctyltrichlorosilane (PFO, Sigma Aldrich, 97\%), fluorescein isothiocyanate (FITC), phosphate-buffered saline (PBS), potassium hydroxide ( $\mathrm{KOH}$, Sigma Aldrich, $\geq 86 \%$ ), erythrosine B (Sigma Aldrich, dye content $\geq 95 \%$ ), potassium iodide (KI, Sigma Aldrich, $\geq 99 \%$ ) and galden perfluoropolyether fluorinated (PFPE) fluid (Blaser Swisslube) were used as received.

\section{Preparation of precursors}

Cesium oleate precursor solution: $\mathrm{Cs}_{2} \mathrm{CO}_{3}(0.204 \mathrm{~g}), \mathrm{ODE}(40 \mathrm{~mL})$ and $\mathrm{OA}(2.5 \mathrm{~mL})$ were placed in a 100 $\mathrm{mL}$ Schlenk flask and dried at $120^{\circ} \mathrm{C}$ under vacuum for 2 hours to allow dissolution of the cesium salt. The solution was then allowed to cool and could then be stored under argon for several days without any observable precipitation at room temperature. For each experiment, $8 \mathrm{~mL}$ of precursor solution was loaded into a $10 \mathrm{~mL}$-gas tight glass syringe (Hamilton). 
Lead halide precursor solution: $\mathrm{PbX}_{2}$ including $\mathrm{PbBr}_{2}(0.069 \mathrm{~g})$ and $\mathrm{PbI}_{2}(0.087 \mathrm{~g})$ were added to $20 \mathrm{~mL}$ Schlenk flasks together with $5 \mathrm{~mL}$ of ODE and dried under vacuum for 2 hours at $120{ }^{\circ} \mathrm{C}$. After this period of time, $0.8 \mathrm{~mL}$ of dried $\mathrm{OA}$ and dried OLA were added under argon until all the $\mathrm{PbX}_{2}$ is completely dissolved. The resulting solutions were then allowed to cool to ambient before being loaded into $10 \mathrm{~mL}$-gas tight glass syringes (Hamilton).

\section{Microfluidic platform and experimental procedure}

Precision syringe pumps (neMESYS, Cetoni GmbH, Germany) were used to inject and motivate the dispersed phase (i.e. $\mathrm{PbX}_{2}$ and Cs-oleate precursor solutions) and carrier fluid (Galden perfluoropolyether fluorinated fluid) towards a PEEK cross (P-729, Upchurch Scientific, Germany) to form a segmented flow of droplets. For $\mathrm{Cs} \mathrm{Pb}(\mathrm{X} / \mathrm{Y})_{3}$, the dispersed $\mathrm{PbX}_{2}$ and $\mathrm{PbY}_{2}$ precursor solutions were initially injected into a PEEK T-junction before being loaded into the PEEK cross for droplet formation. The injection cross and the syringes carrying the precursor solutions where connected through PTFE tubing (ID $250 \mu \mathrm{m}$, OD 1/16", Upchurch Scientific, Germany) using PEEK fingertight fittings (F-127, Upchurch Scientific, Germany). The carrier fluid was transferred to the PEEK cross through fluorinated ethylene propylene tubing (ID 750 $\mu \mathrm{m}$, OD 1/16", Upchurch Scientific, Germany). Typical flow rates for the carrier phase were between 50 and $120 \mu \mathrm{L} / \mathrm{min}$ and between 0.5 and $30 \mu \mathrm{L} / \mathrm{min}$ for the dispersed phase. As previously reported, the microfluidic platform allowed for independent control of the Pb-to-Cs molar ratio and halide ratios (I-to$\mathrm{Br}$ ) through adjustment of flow rates ratios at the cross and T-junctions, respectively. The formed droplets containing the reaction mixture were subsequently directed through perfluoroalkoxy alkane (PFA) tubing (ID $500 \mu \mathrm{m}$, OD 1/16", Upchurch Scientific, Germany) coiled around a copper-heating rod (diameter $=1.5$ $\mathrm{cm}$ ) to allow the initiation of reaction. Droplets exiting the heating section were directed through PTFE tubing into a straight channel $(500 \times 80 \mu \mathrm{m})$ polydimethylsiloxane (PDMS) microfluidic flow cell to allow for concurrent time-integrated and time-resolved measurements. The PDMS microfluidic device was fabricated using standard soft-lithographic techniques, which are described elsewhere. ${ }^{90}$ Surface treatment of the microfluidic channels with PFO was performed prior to each experiment to ensure that channel surfaces were consistently fluorophilic.

\section{Confocal fluorescence detection}

For all TCSPC experiments, light from a $485 \mathrm{~nm}$ pulsed diode laser (P-C-485 $390 \mathrm{ps}$ pulse width, PicoQuant $\mathrm{GmbH}$, Germany) was directed towards a laser clean-up filter (F1) (F49-488, Chroma, Germany) and then coupled by a fiber delivery system $(F C)$ using a single mode fiber (KineFLEX-P-3-S-488, QIOPTIQ, Germany). Light exiting the fiber was then collimated by an infinity-corrected 10x magnification microscope objective (Olympus 10x NA 0.25, Thorlabs, Germany) for laser beam expansion and directed into the back port of the microscope (Nikon Ti-E). Expansion of the laser beam ensured that the beam almost 


\section{In-situ PL measurements and estimation of fluorescence lifetimes}

In-situ PL intensity and lifetime measurements of $\mathrm{CsPb}(\mathrm{X} / \mathrm{Y})_{3} \mathrm{NCs}$ were recorded from individual droplets travelling along the PDMS microfluidic channel. PL data were recorded with a $100 \mathrm{~ms}$ integration time using a fiber spectrometer (Qe Pro, Ocean Optics, UK) through a $2 \mathrm{~m}$ long fiber mounted to one of the ports of the microscope. TCSPC measurements were recorded with a 50-100 ms integration time, $0.025 \mathrm{~ns}$ binning time and $10 \mathrm{~s}$ total acquisition time, by further averaging over multiple droplets. In-house software (written in the LabView programming environment) was used to calculate real-time fluorescence decay times of the synthesized NCs, with decay profiles being fitted to a sum-of-exponential models containing up to three terms, Eq. 3. The fluorescence decay measured by the TCSPC method corresponds to the convolution of the instrument response function (IRF) of the microscope with the actual fluorescence decay of the sample. Since the IRF affects the accuracy of measured fluorescence lifetime, we measure its value by an IRF deconvolution procedure to improve the accuracy of the lifetime measurement. The solution for the IRF measurement was prepared by the mixing $170 \mu \mathrm{L}$ of saturated Erythrosine B solution with $300 \mu \mathrm{L} 0.004 \mathrm{M}$ $\mathrm{KOH}$ solution in $1 \mathrm{~mL}$ water solution containing saturated KI.

$$
I(t)=I_{0} \sum_{i=1}^{n} \exp \left(\frac{t}{\tau_{i}}\right)
$$


Here $I(t)$ is intensity at time $t, I_{0}$ is the intensity at time zero and $\tau_{i}$ is the lifetime of the $i^{\text {th }}$ component. The average lifetime $(\tau)$ was calculated using Eq. 4, with parameter estimation being performed in an iterative fashion by minimization of the reduced chi-squared parameter, i.e.

$$
\tau=\frac{\sum_{i=1}^{n}\left(a_{i} \tau_{i}^{2}\right)}{\sum_{i=1}^{n}\left(a_{i} \tau_{i}\right)} \quad \sum a_{i}=1
$$

Here, $a_{i}$ is the amplitude (or pre-exponential factor) associated with component $i$, where $\sum a_{i}=1$. A Levenberg-Marquardt minimization algorithm was used to fit all data, and a Richardson-Lucy algorithm used for the deconvolution of the IRF from recorded decay profiles. The analysis software allows control of the number of fitting parameters, with the initial parameter estimates being provided manually at the start the fitting procedure. Fitting of the fluorescence decays originating from the synthesized NCs (within individual droplets) can be performed in high-throughput, with the average fluorescence lifetime being calculated in an automatic manner (see Figure S1 for single droplet fluorescence lifetime analysis).

It is important to note that the reactor operation is entirely automated and is also controlled by a custom made LabView script. Briefly, the LabView script accepts a set of predefined experimental parameters that are executed in sequence. First, the reactor checks current reaction conditions (such as temperature and volumetric flow rates) and then determines an appropriate time-scale for the system to reach steady-state. Reaction conditions are then adjusted and after measurement of fluorescence decay curves, fluorescence decay times are extracted. Figure 2 summarizes the workflow for this process.

\section{Results and Discussion}

To calibrate the real-time TCSPC detection system and assess the robustness of the analysis procedure, we measured the fluorescence decay of a $100 \mu \mathrm{M}$ fluorescein isothiocyanate (FITC) solution in PBS buffer enclosed into nanoliter droplets. The fluorescence decay profile is shown in Figure 3, and is satisfactorily described by a single exponential decay function with a fluorescence lifetime of $4.18 \mathrm{~ns}$ and a reduced chisquared value of 1.23 .

Colloidal $\mathrm{CsPbX}_{3} \mathrm{NCs}$ were then synthesized according to procedures described in our recent publication. ${ }^{46}$ As previously elucidated, the molar ratio of $\mathrm{Pb}$-to-Cs should be maintained above 1.5 to ensure that the synthesis can proceed in the stoichiometric fashion with full reaction yield (forming $\mathrm{Pb}$-oleate as a byproduct). ${ }^{46}$ In particular, multiple variations of $\mathrm{Pb}$-to-Cs reagent ratios can have a dramatic effect on $\mathrm{PL}$ properties, such as fwhm and PL intensity. Additionally, $\mathrm{Pb}$ rich conditions ( $\mathrm{Pb}$-to-Cs molar ratios between 2 and 5, depending on the halide composition) can be beneficial for the formation of $\mathrm{CsPbX}_{3} \mathrm{NCs}$ with enhanced PL QYs. ${ }^{46}$ To further understand the effect of the particle concentration on time-integrated 
For instance, Figure $4 \mathrm{c}$ illustrates that an increase of the $\mathrm{Pb}$-to-Cs molar ratio from 1.7 to 3.1 leads to an increase of the average fluorescence lifetime from 17.2 to $19.2 \mathrm{~ns}$, whereas a further increase of $\mathrm{Pb}$-to-Cs ratio causes a drop to $13.5 \mathrm{~ns}$ probably associated to the nonradiative recombination. The average lifetime of all halide compositions tested, has a strong dependence on Pb-to-Cs variations (see Supporting Information for additional studies of $\mathrm{Pb}$-to-Cs variations at different temperatures, Figures S2-S4), particularly for $\mathrm{CsPb}_{3}$ where the average lifetime drops from 44 to $15 \mathrm{~ns}$ for Pb-rich NCs.

To obtain additional insights into the role of NC compositional changes on the PL properties, we also measured the fluorescence lifetimes of $\mathrm{CsPb}(\mathrm{Br} / \mathrm{I})_{3} \mathrm{NCs}$ while varying the halide composition. As shown in our recent study, ${ }^{46}$ the band edge emission of Br/I perovskites can be tuned from 523 to $690 \mathrm{~nm}$ through a continuous variation of $\mathrm{PbI}_{2} / \mathrm{PbBr}_{2}$ halide molar ratio (supporting information for PL spectra). In general, PL lifetime is both composition and size-dependent at room temperature. Specifically, for larger bandgaps - smaller NC sizes or lighter halides - the PL lifetime is shorter. ${ }^{55}$ Such bandgap-dependence of the radiative rate is fully explainable by the Fermi golden rule, also known as the energy gap law. ${ }^{94}$ In this direction, adjustment of the anionic composition of the synthesized $\mathrm{CsPbX}_{3} \mathrm{NCs}$ serves two purposes: it provides a facile manner to tune the PL properties of the perovskite NCs and at the same time possible size-dependent effects on the measured PL lifetimes can be excluded when the size of $\mathrm{CsPbX}_{3}$-based NCs is fixed between 10-12 nm (e.g. within the weak quantum confinement regime). ${ }^{59,66}$ Figure 5a-b demonstrates the effect of the halide content on the normalized fluorescence decays of $\mathrm{CsPb}(\mathrm{Br} / \mathrm{I})_{3} \mathrm{NCs}$. Extraction of the average fluorescence lifetimes from the fluorescence decay data were plotted as a function of iodide content and showed a practically linear increase. 
Previously, we demonstrated that the band-edge emission and the $\mathrm{PL}$ decays of $\mathrm{CsPb}(\mathrm{Br} / \mathrm{I})_{3}$ can be tuned as a function of $\mathrm{I} / \mathrm{Br}$ molar ratio. ${ }^{46}$ Therefore, by a systematic variation of iodide loading from 6.5 to $91.6 \%$, we managed to tune the PL peak wavelength from 516 to $680 \mathrm{~nm}$ (Figure $5 \mathrm{c}$ ) leading to a monotonic increase of average PL lifetimes from 12.5 (Br-rich) to $30 \mathrm{~ns}$ (I-rich) (Figure 5b). This confirms that the PL lifetime is strongly dependent on the halide confirming that the heavier is the halide, the longer is the PL lifetime. However, the long PL lifetime could be attributed to the formation of metastable charge-separated states involving electrons in shallow surface traps. ${ }^{91}$

To further investigate the effect of iodide content on the fluorescence decays, we conducted experiments at various temperatures with a fixed $\mathrm{Pb}$-to-Cs molar ratio. Figure $5 \mathrm{~d}$ demonstrates the effect of iodide loading from 6.5 to $91.6 \%$ on the average lifetime of the synthesized NCs, at 150,170 and $180{ }^{\circ} \mathrm{C}$ (temperatures correspond to the synthesis of NCs - lifetime measurements were conducted at room temperature, after droplets exiting the reactor). Although fluorescence lifetimes tend to increase at all temperatures, however at iodide loadings between 25 and 50\%, they remain mostly constant or slightly decrease. This can be explained by the fact that at this range of iodide loading a drop in PL intensity is observed, mostly due to the fast halide-ion-segregation during the formation of $\mathrm{CsPb}(\mathrm{Br} / \mathrm{I})_{3} \mathrm{NCs}^{61}$

Although, variation of the anionic composition towards the formation of Br-rich and I-rich perovskite NCs provides a facile tuning of their PL spectra from blue to red, a concurrent readjustment of $\mathrm{Pb}$-to-Cs molar needs to be considered. ${ }^{46}$ For this reason, we performed experiments at different $\mathrm{Pb}$-to-Cs molar ratios (which has a strong influence on fwhm and emission intensity of $\mathrm{CsPb}(\mathrm{Br} / \mathrm{I})_{3}$ while altering the anionic composition of the NCs (see Supporting Information, Figures S5-S6). In general, Pb-to-Cs molar ratios in the range of 2.2-3.1 allowed for the tuning of the PL lifetimes between 12-32 ns from Br-rich to I-rich compositions (Figure 5 and Figure S5). On the other hand, $\mathrm{Pb}$-to-Cs molar ratios greater than 4 do not favor the formation of luminescent Br-rich mixed halide NCs, since the fluorescence lifetime decays of NCs with iodide compositions $\leq 50 \%$ could not be detected ( see Figure $\mathrm{S} 6, \mathrm{~Pb}$-to-Cs $=4.1$ ). At the same time, average lifetimes of I-rich NCs were considerably lower than those synthesized at lower Pb-to-Cs molar ratios. These findings further support our previous study of different optimized $\mathrm{Pb}$-to-Cs molar ratios for the formation of highly luminescent Br-rich and I-rich $\mathrm{CsPb}(\mathrm{Br} / \mathrm{I})_{3} \mathrm{NCs}^{46}$

Typical flask syntheses $\mathrm{CsPbX}_{3} \mathrm{NCs}$ are conducted between 160 and $180^{\circ} \mathrm{C}$ for obtaining sizes in the range of 8-12 nm. ${ }^{2}$ As it has been reported, ${ }^{2}$ an optimum range of reaction temperatures for the synthesis of $\mathrm{CsPbX}$ NCs, which exhibit high QYs (up to 90\%) and narrow emission linewidths, is between 130 and $200{ }^{\circ} \mathrm{C}$. In this respect, such high QYs are relatively uncommon for NCs when synthesized at relatively low temperatures. To extend the optical characterization of $\mathrm{CsPbX}_{3} \mathrm{NCs}$, we investigated the fluorescence lifetime behavior of $\mathrm{CsPbX}_{3} \mathrm{NCs}$ as a function of temperature for reaction times of at least $10 \mathrm{~s}$. Figure 6a- 


\section{Conclusion}

The studies described represent the very first report of on-the-fly TCSPC measurements during the synthesis of perovskite NCs and suggest significant potential for the rapid optimization of NC photophysics using minimal reagent volumes $\left(10^{6}\right.$-fold reduction in sample volume compared to conventional analysis) and reduced screening times ( $\sim 200$ experimental runs per hour meaning 100-fold reduction in terms of screening times). In addition, concurrent time-integrated PL and TCSPC measurements revealed that intensity variations from PL measurements were not an effect of product yield but directly proportional to average lifetime. In addition, a thorough investigation on the influence of different precursors ( $\mathrm{Pb}$-to- $\mathrm{Cs}$ and $\mathrm{Br} / \mathrm{I}$ ratios) and temperature on the average lifetimes allowed us to verify our conclusions regarding the optimized ratios required for the synthesis of highly luminescent $\mathrm{CsPbX}_{3} \mathrm{NCs}$. In particular, systematic variation of reaction conditions allowed a fine tuning of the average lifetime between 5 and 42 ns. Importantly, the average lifetime is strongly dependent on the halide content. An increase in the iodide content, for mixed halide perovskites, allowed for a linear increase of the fluorescence lifetimes. However, long PL lifetimes could be attributed to the formation of metastable charge-separated states involving electrons in shallow surface traps or polarons. In addition, such platforms are ideally suited for the rapid determination of radiative multinary compositions (such as $\mathrm{CuZnIn}(\mathrm{Se} / \mathrm{S})_{4}$ and $\left.\mathrm{Cu}(\mathrm{Ga} / \mathrm{In})(\mathrm{S} / \mathrm{Se})_{4}\right)$ whilst providing mechanistic insights based on intrinsic photophysical properties. A refined version of this platform can potentially track the lifetimes of NCs at the early stages of their formation. Elucidating the nucleation and growth mechanism of semiconductor nanocrystals in the millisecond-timescale remains challenging. Droplet-based microfluidics integrated with fluorescence lifetime measurements will allow extraction of fluorescence decay times and spectra at early-time points during the nucleation growth processes. Experiments toward this goal are in progress. Other applications include protein screening for the extraction of a wide range of parameters (including $\mathrm{pH}$, temperature and thermodynamics) and fluorescence lifetimeactivated droplet sorting. We anticipate that the core methodology will be of immense value to the nanomaterials and microfluidic communities.

\section{Author Contributions}


The manuscript was written through contributions of all authors. All authors have given approval to the final version of the manuscript.The authors declare no competing financial interest.

\section{Acknowledgements}

The authors would like to thank Dr. Gabriele Raino, Dr. Hendrik Utzat and Dr. Brent Koscher for discussions and recommendations. This work was partially supported by the Swiss Federal Institute of Technology (ETH Zürich) and by a Swiss National Foundation Grant P2EZP2_17212.

\section{Supporting Information}

This material is available free of charge via the internet at http://pubs.acs.org.

Software snaphsot of fluorescence lifetime analysis, the effect of $\mathrm{Pb}$-to-Cs molar ratio on the fluorescence lifetimes of $\mathrm{CsPbI}_{3}$ and $\mathrm{CsPbBr}_{3}$ NCs at various temperatures, the effect of iodide loading on the fluorescence lifetimes of $\mathrm{CsPb}(\mathrm{Br} / \mathrm{I})_{3} \mathrm{NCs}$ at different $\mathrm{Pb}$-to-Cs molar ratios, and the effect of temperature on $\mathrm{PL}$ intensity and average lifetimes of $\mathrm{CsPbBr}_{3}$ and $\mathrm{CsPb}(\mathrm{Br} / \mathrm{I})_{3} \mathrm{NCs}$. 
a



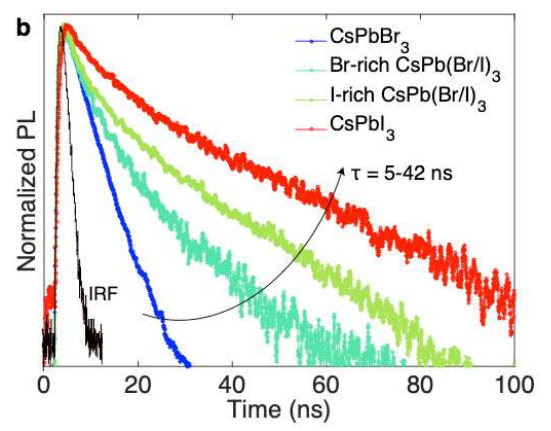

Figure 1: (a) A schematic of the microfluidic platform incorporating a TCSPC module. (b) Representative in-situ PL decays of $\mathrm{Cs} P b X_{3} \mathrm{NCs}$ and the instrument response function (IRF). Radiative lifetimes were estimated using a multiexponential fitting function in real-time. 


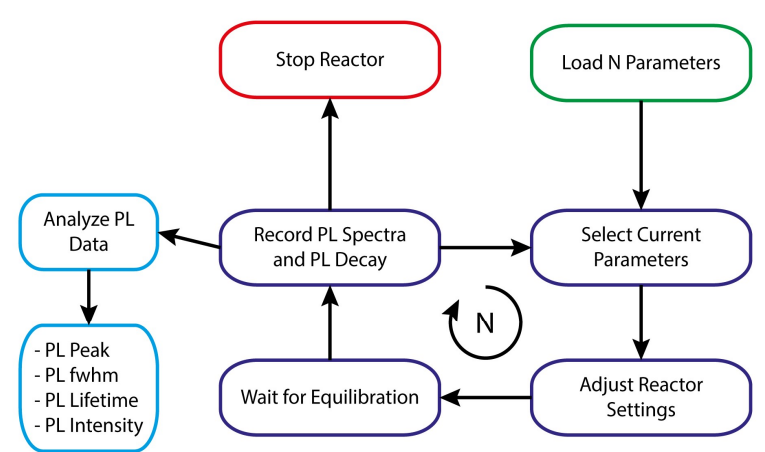

Figure 2: Flow chart for the automated operation of the microfluidic platform and recording of PL lifetimes and PL spectra. 

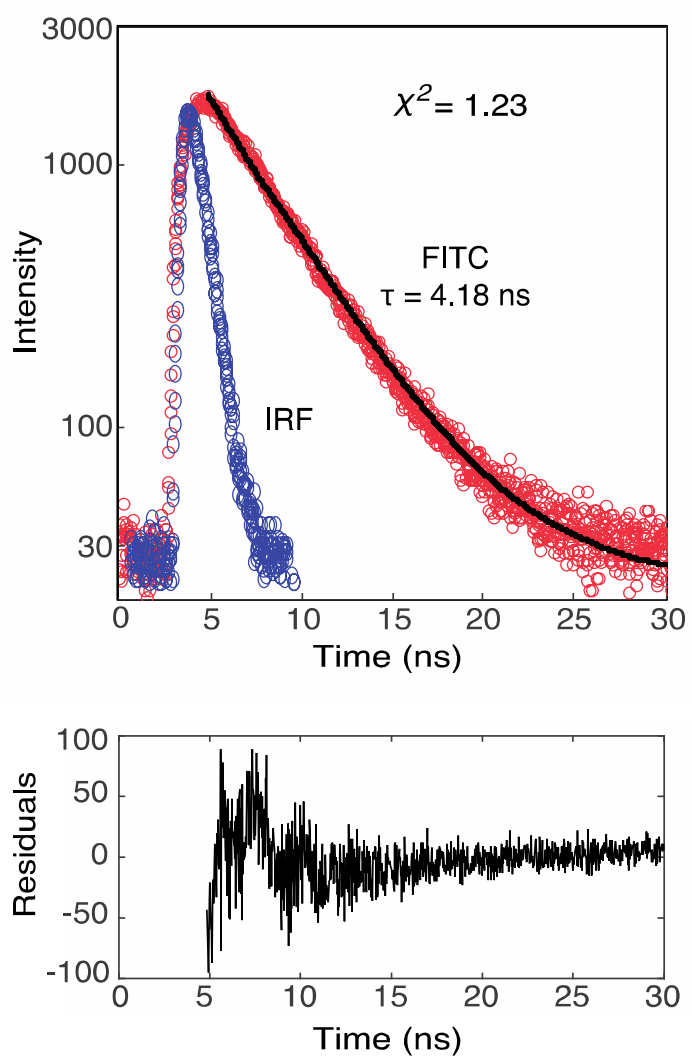

Figure 3: Fluorescence lifetime analysis of $100 \mu M$ FITC solution in PBS buffer. Other parameters were: $\lambda_{e x}=488 \mathrm{~nm}$, pulse width $=80 \mathrm{ps}$ and repetition rate $=20 \mathrm{MHz}$. 

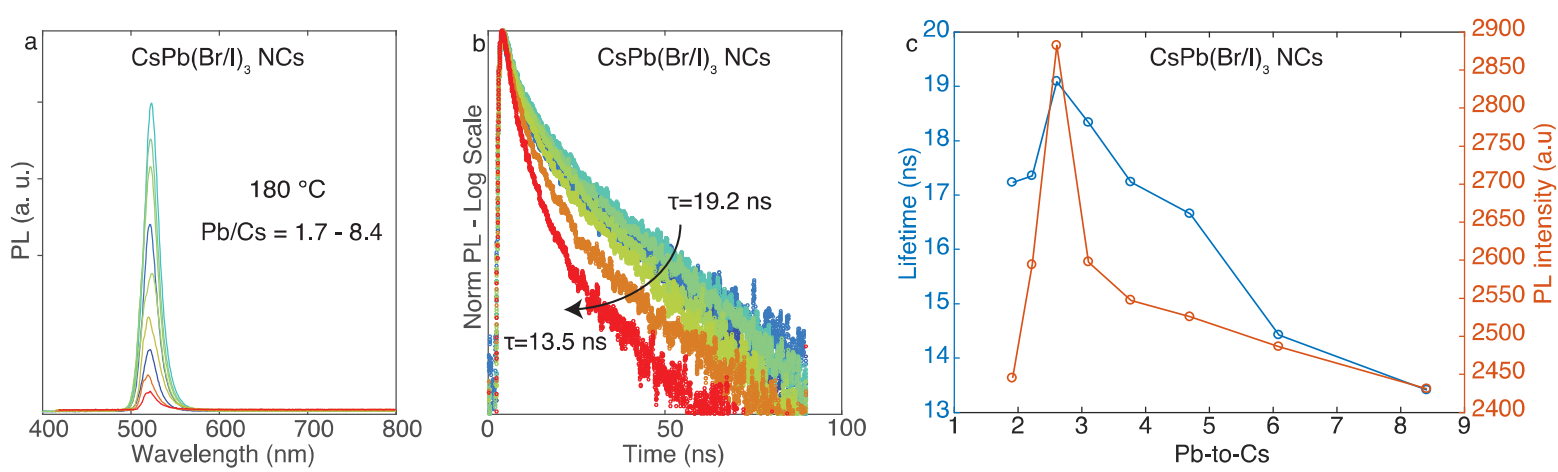

Figure 4: The effect of the Pb-to-Cs molar ratio on the (a) PL characteristics and (b) normalized fluorescence decays (log-scale) of $\mathrm{Cs} P b(\mathrm{Br} / \mathrm{I})_{3} \mathrm{NCs}$ at $180^{\circ} \mathrm{C}$. Colors in the PL spectra correspond to various $\mathrm{Pb} / \mathrm{Cs}$ values. The PL decays were fitted using a biexponential model and they are extracted after averaging over multiple droplets with the same experimental conditions. (c) Pbto-Cs molar ratios vs $\tau$ and $P L$ intensity for the synthesized $\mathrm{Cs} P b(\mathrm{Br} / \mathrm{I})_{3} \mathrm{NCs}$. Other reaction conditions were fixed to reaction time $=10 \mathrm{~s}$ and iodide loading $=16 \%$. 
a
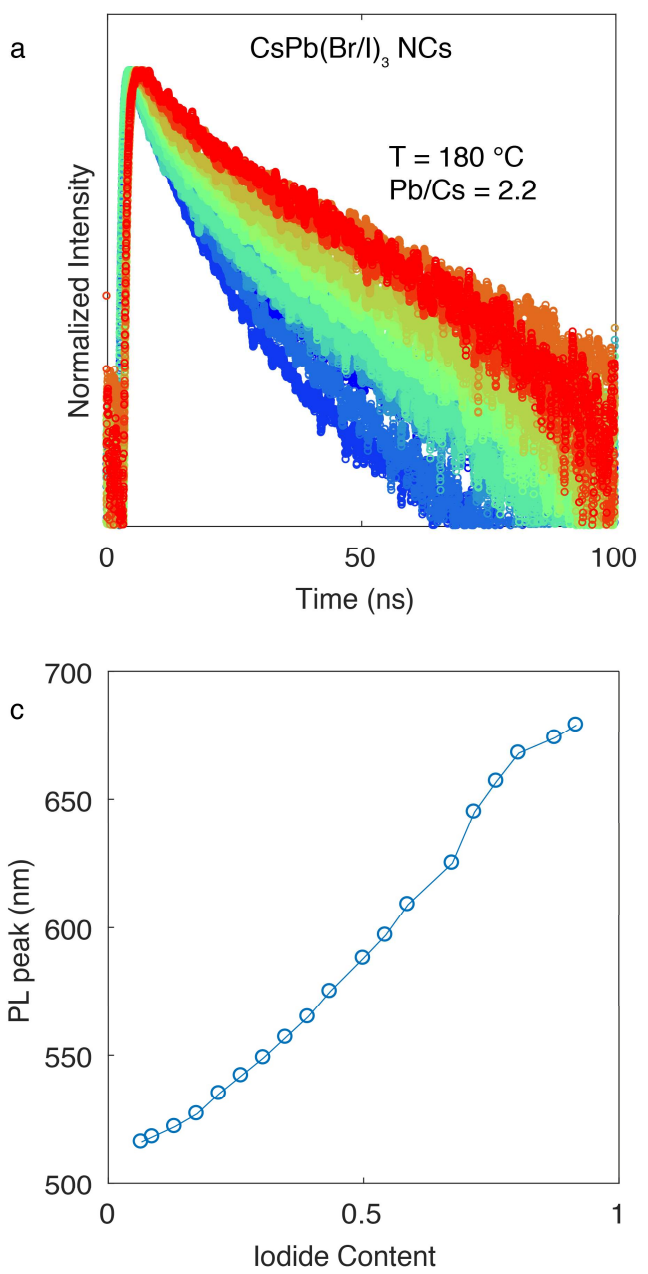
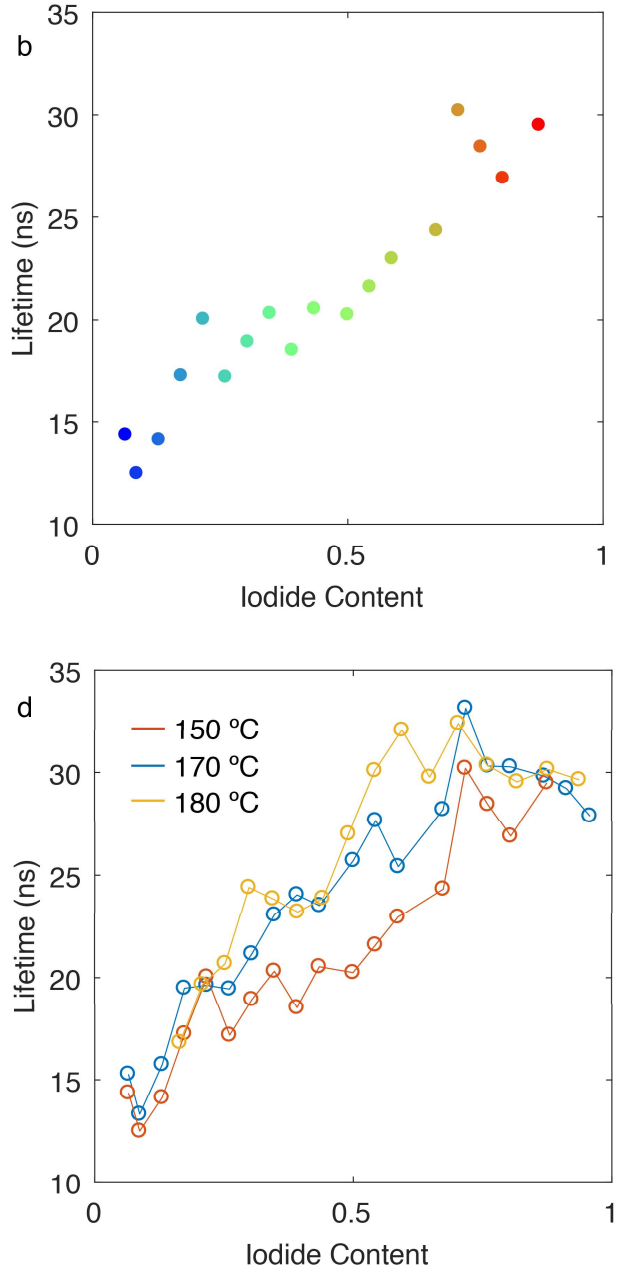

Figure 5: The effect of iodide loading on the (a) normalized fluorescence decays (log-scale),(b) average lifetimes ( $\tau$ ), (c) position of maximum emission of $\mathrm{Cs} \mathrm{Pb}(\mathrm{Br} / \mathrm{I})_{3} \mathrm{NCs}$ at $170^{\circ} \mathrm{C}$.Colors in Figure $5 \mathrm{~b}$ correspond to the fluorescence decays in Figure 5 a. The $P L$ decays were fitted using a biexponential model and they are extracted after averaging over multiple droplets with the same experimental conditions. (d) Iodide content vs average lifetime for $\mathrm{Cs} P b(\mathrm{Br} / \mathrm{I})_{3} \mathrm{NCs}$ which were synthesized at various temperatures 150,170 and $180^{\circ} \mathrm{C}$. Other reaction conditions were fixed to $\mathrm{Pb}$-to-Cs $=2.2$ and reaction time $=10 \mathrm{~s}$. 

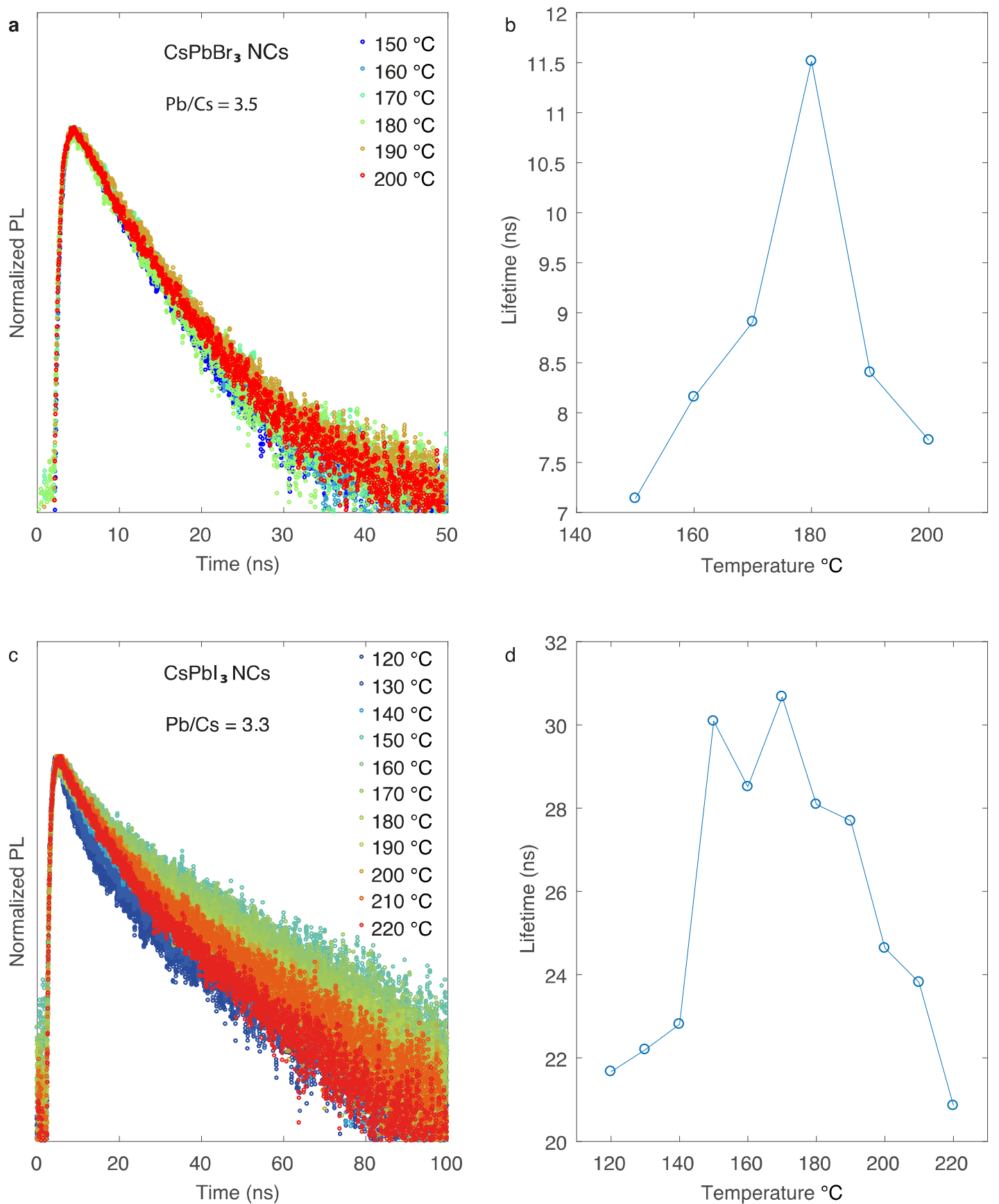

Figure 6: The effect of temperature on the ( $a, c)$ normalized fluorescence decays (log-scale) and $(b, d)$ average lifetimes $(\tau)$ of $\mathrm{Cs}_{\mathrm{PbBr}}$ and $\mathrm{Cs}_{\mathrm{PbI}} \mathrm{NCs}$. The PL decays were fitted using a biexponential model and they are extracted after averaging over multiple droplets with the same experimental conditions. Other reaction conditions were fixed to $\mathrm{Pb}$-to-Cs $=3.5$ for $\mathrm{Cs} P b B r_{3} \mathrm{NCs}$, $\mathrm{Pb}$-to-Cs $=3.3$ for $\mathrm{Cs} \mathrm{PbI}_{3} \mathrm{NCs}$ and reaction time $=10 \mathrm{~s}$. 


\section{Graphical TOC Entry}

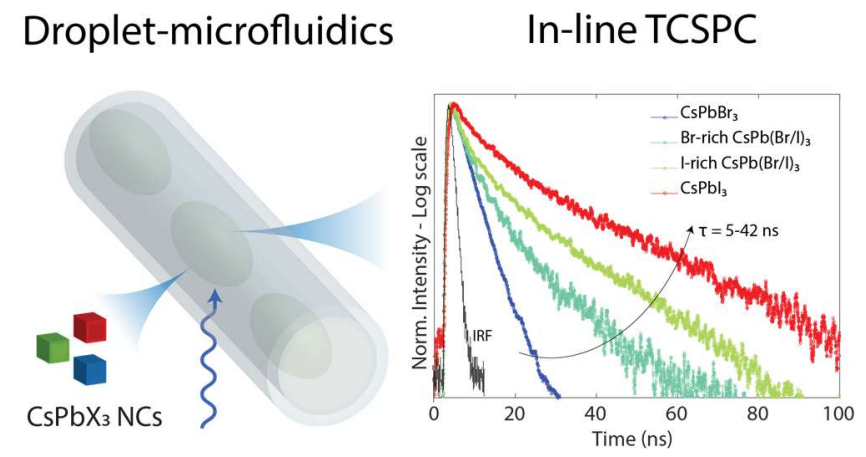




\section{References}

1. Pietryga, J. M.; Park, Y.-S.; Lim, J.; Fidler, A. F.; Bae, W. K.; Brovelli, S.; Klimov, V. I., Spectroscopic and Device Aspects of Nanocrystal Quantum Dots. Chem. Rev. 2016, 116 (18), 10513-10622.

2. Protesescu, L.; Yakunin, S.; Bodnarchuk, M. I.; Krieg, F.; Caputo, R.; Hendon, C. H.; Yang, R. X.; Walsh, A.; Kovalenko, M. V., Nanocrystals of Cesium Lead Halide Perovskites (CsPbX3, X = Cl, Br, and I): Novel Optoelectronic Materials Showing Bright Emission with Wide Color Gamut. Nano Lett. 2015, 15 (6), 3692-3696.

3. Howes, P. D.; Chandrawati, R.; Stevens, M. M., Colloidal nanoparticles as advanced biological sensors. Science 2014, 346 (6205), 1247390-1247390.

4. $\quad$ Frecker, T.; Bailey, D.; Arzeta-Ferrer, X.; McBride, J.; Rosenthal, S. J., Review - Quantum Dots and Their Application in Lighting, Displays, and Biology. ECS J. Solid State Sci. Technol. 2016, 5 (1), R3019-R3031.

5. Wood, V.; Bulović, V., Colloidal quantum dot light-emitting devices. Nano Rev. 2010, 1, 5202-5202.

6. Chiba, T.; Hoshi, K.; Pu, Y. J.; Takeda, Y.; Hayashi, Y.; Ohisa, S.; Kawata, S.; Kido, J., High-Efficiency Perovskite Quantum-Dot Light-Emitting Devices by Effective Washing Process and Interfacial Energy Level Alignment. ACS Appl. Mater. Interfaces 2017, 9 (21), 18054-18060.

7. $\quad$ Deng, W.; Xu, X. Z.; Zhang, X. J.; Zhang, Y. D.; Jin, X. C.; Wang, L.; Lee, S. T.; Jie, J. S., Organometal Halide Perovskite Quantum Dot Light-Emitting Diodes. Adv. Funct. Mater. 2016, 26 (26), 4797-4802.

8. Li, G. R.; Rivarola, F. W. R.; Davis, N. J. L. K.; Bai, S.; Jellicoe, T. C.; de la Pena, F.; Hou, S. C.; Ducati, C.; Gao, F.; Friend, R. H.; Greenham, N. C.; Tan, Z. K., Highly Efficient Perovskite Nanocrystal Light-Emitting Diodes Enabled by a Universal Crosslinking Method. Adv. Mater. 2016, 28 (18), 3528-+.

9. Li, J. H.; Xu, L. M.; Wang, T.; Song, J. Z.; Chen, J. W.; Xue, J.; Dong, Y. H.; Cai, B.; Shan, Q. S.; Han, B. N.; Zeng, H. B., 50-Fold EQE Improvement up to $6.27 \%$ of Solution-Processed All-Inorganic Perovskite CsPbBr3 QLEDs via Surface Ligand Density Control. Adv. Mater. 2017, 29 (5).

10. Pan, J.; Quan, L. N.; Zhao, Y.; Peng, W.; Murali, B.; Sarmah, S. P.; Yuan, M.; Sinatra, L.; Alyami, N. M.; Liu, J.; Yassitepe, E.; Yang, Z.; Voznyy, O.; Comin, R.; Hedhili, M. N.; Mohammed, O. F.; Lu, Z. H.; Kim, D. H.; Sargent, E. H.; Bakr, O. M., Highly Efficient Perovskite-Quantum-Dot Light-Emitting Diodes by Surface Engineering. Adv. Mater. 2016, 28 (39), 8718-8725.

11. Tan, Y. S.; Zou, Y. T.; Wu, L. Z.; Huang, Q.; Yang, D.; Chen, M.; Ban, M. Y.; Wu, C.; Wu, T.; Bai, S.; Song, T.; Zhang, Q.; Sun, B. Q., Highly Luminescent and Stable Perovskite Nanocrystals with Octylphosphonic Acid as a Ligand for Efficient Light-Emitting Diodes. ACS Appl. Mater. Interfaces 2018, 10 (4), 3784-3792.

12. Zhang, X. Y.; Sun, C.; Zhang, Y.; Wu, H.; Ji, C. Y.; Chuai, Y. H.; Wang, P.; Wen, S. P.; Zhang, C. F.; Yu, W. W., Bright Perovskite Nanocrystal Films for Efficient Light-Emitting Devices. J. Phys. Chem. Lett. 2016, 7 (22), 4602-4610.

13. Konstantatos, G.; Sargent, E. H., Nanostructured materials for photon detection. Nat. Nanotechnol. 2010, 5 (6), 391-400.

14. Tang, X.; Ackerman, M. M.; Chen, M. L.; Guyot-Sionnest, P., Dual-band infrared imaging using stacked colloidal quantum dot photodiodes. Nat. Photonics 2019, 13 (4), 277-282.

15. Ramasamy, P.; Lim, D. H.; Kim, B.; Lee, S. H.; Lee, M. S.; Lee, J. S., All-inorganic cesium lead halide perovskite nanocrystals for photodetector applications. Chem. Commun. 2016, 52 (10), 2067-2070.

16. Gong, M. G.; Sakidja, R.; Goul, R.; Ewing, D.; Casper, M.; Stramel, A.; Elliot, A.; Wu, J. Z., HighPerformance All-Inorganic CsPbCl3 Perovskite Nanocrystal Photodetectors with Superior Stability. Acs Nano 2019, $13(2), 1772-1783$.

17. Kovalenko, M. V., Opportunities and challenges for quantum dot photovoltaics. Nat. Nanotechnol. 2015, 10 (12), 994-997.

18. Wang, X. H.; Koleilat, G. I.; Tang, J.; Liu, H.; Kramer, I. J.; Debnath, R.; Brzozowski, L.; Barkhouse, D. A. R.; Levina, L.; Hoogland, S.; Sargent, E. H., Tandem colloidal quantum dot solar cells employing a graded recombination layer. Nat. Photonics 2011, 5 (8), 480-484.

19. Sanehira, E. M.; Marshall, A. R.; Christians, J. A.; Harvey, S. P.; Ciesielski, P. N.; Wheeler, L. M.; Schulz, P.; Lin, L. Y.; Beard, M. C.; Luther, J. M., Enhanced mobility CsPbI3 quantum dot arrays for record-efficiency, highvoltage photovoltaic cells. Sci. Adv. 2017, 3 (10), eaao4204.

20. Swarnkar, A.; Marshall, A. R.; Sanehira, E. M.; Chernomordik, B. D.; Moore, D. T.; Christians, J. A.; Chakrabarti, T.; Luther, J. M., Quantum dot-induced phase stabilization of alpha-CsPbI3 perovskite for high-efficiency photovoltaics. Science 2016, 354 (6308), 92-95.

21. Wheeler, L. M.; Sanehira, E. M.; Marshall, A. R.; Schulz, P.; Suri, M.; Anderson, N. C.; Christians, J. A.; Nordlund, D.; Sokaras, D.; Kroll, T.; Harvey, S. P.; Berry, J. J.; Lin, L. Y.; Luther, J. M., Targeted Ligand-Exchange Chemistry on Cesium Lead Halide Perovskite Quantum Dots for High-Efficiency Photovoltaics. J. Am. Chem. Soc. 2018, 140 (33), 10504-10513.

22. Kagan, C. R.; Lifshitz, E.; Sargent, E. H.; Talapin, D. V., Building devices from colloidal quantum dots. Science 2016, 353 (6302), 885-885. 
23. Kovalenko, M. V.; Manna, L.; Cabot, A.; Hens, Z.; Talapin, D. V.; Kagan, C. R.; Klimov, V. I.; Rogach, A. L.; Reiss, P.; Milliron, D. J.; Guyot-Sionnnest, P.; Konstantatos, G.; Parak, W. J.; Hyeon, T.; Korgel, B. A.; Murray, C. B.; Heiss, W., Prospects of nanoscience with nanocrystals. ACS Nano 2015, 9 (2), 1012-1057.

24. Lakowicz, J. R., Principles offluorescence spectroscopy. 3rd ed.; Springer: New York, 2006.

25. Sutherland, B. R.; Sargent, E. H., Perovskite photonic sources. Nat. Photonics 2016, 10 (5), 295-302.

26. Kovalenko, M. V.; Protesescu, L.; Bodnarchuk, M. I., Properties and potential optoelectronic applications of lead halide perovskite nanocrystals. Science 2017, 358 (6364), 745-750.

27. Akkerman, Q. A.; Raino, G.; Kovalenko, M. V.; Manna, L., Genesis, challenges and opportunities for colloidal lead halide perovskite nanocrystals. Nat. Mater. 2018, 17 (5), 394-405.

28. Song, J. Z.; Li, J. H.; Li, X. M.; Xu, L. M.; Dong, Y. H.; Zeng, H. B., Quantum Dot Light-Emitting Diodes Based on Inorganic Perovskite Cesium Lead Halides (CsPbX3). Adv. Mater. 2015, 27 (44), 7162-7167.

29. Li, X. M.; Wu, Y.; Zhang, S. L.; Cai, B.; Gu, Y.; Song, J. Z.; Zeng, H. B., CsPbX3 Quantum Dots for Lighting and Displays: Room-Temperature Synthesis, Photoluminescence Superiorities, Underlying Origins and White LightEmitting Diodes. Adv. Funct. Mater. 2016, 26 (15), 2435-2445.

30. Huang, H.; Polavarapu, L.; Sichert, J. A.; Susha, A. S.; Urban, A. S.; Rogach, A. L., Colloidal lead halide perovskite nanocrystals: synthesis, optical properties and applications. Npg Asia Materials 2016, 8, e328.

31. Lignos, I.; Morad, V.; Shynkarenko, Y.; Bernasconi, C.; Maceiczyk, R. M.; Protesescu, L.; Bertolotti, F.; Kumar, S.; Ochsenbein, S. T.; Masciocchi, N.; Guagliardi, A.; Shih, C. J.; Bodnarchuk, M. I.; deMello, A. J.; Kovalenko, M. V., Exploration of Near-Infrared-Emissive Colloidal Multinary Lead Halide Perovskite Nanocrystals Using an Automated Microfluidic Platform. ACS Nano 2018, 12 (6), 5504-5517.

32. Swarnkar, A.; Chulliyil, R.; Ravi, V. K.; Irfanullah, M.; Chowdhury, A.; Nag, A., Colloidal CsPbBr3 Perovskite Nanocrystals: Luminescence beyond Traditional Quantum Dots. Angew. Chem. Int. Ed. 2015, 54 (51), 15424-15428.

33. Tong, Y.; Bladt, E.; Ayguler, M. F.; Manzi, A.; Milowska, K. Z.; Hintermayr, V. A.; Docampo, P.; Bals, S.; Urban, A. S.; Polavarapu, L.; Feldmann, J., Highly Luminescent Cesium Lead Halide Perovskite Nanocrystals with Tunable Composition and Thickness by Ultrasonication. Angew. Chem. Int. Ed. 2016, 55 (44), 13887-13892.

34. Akkerman, Q. A.; Gandini, M.; Di Stasio, F.; Rastogi, P.; Palazon, F.; Bertoni, G.; Ball, J. M.; Prato, M.; Petrozza, A.; Manna, L., Strongly emissive perovskite nanocrystal inks for high-voltage solar cells. Nat. Energy 2016, 2, 16194-16194.

35. Dutta, A.; Pradhan, N., Phase-Stable Red-Emitting CsPbI3 Nanocrystals: Successes and Challenges. ACS Energy Lett. 2019, 4 (3), 709-719.

36. Dutta, A.; Dutta, S. K.; Das Adhikari, S.; Pradhan, N., Tuning the Size of CsPbBr3 Nanocrystals: All at One Constant Temperature. ACS Energy Lett. 2018, 3 (2), 329-334.

37. Krieg, F.; Ochsenbein, S. T.; Yakunin, S.; ten Brinck, S.; Aellen, P.; Suess, A.; Clerc, B.; Guggisberg, D.; Nazarenko, O.; Shynkarenko, Y.; Kumar, S.; Shih, C. J.; Infante, I.; Kovalenko, M. V., Colloidal CsPbX3 (X = CI, Br, I) Nanocrystals 2.0: Zwitterionic Capping Ligands for Improved Durability and Stability. ACS Energy Lett. 2018, 3 (3), 641-646.

38. Imran, M.; Caligiuri, V.; Wang, M. J.; Goldoni, L.; Prato, M.; Krahne, R.; De Trizio, L.; Manna, L., Benzoyl Halides as Alternative Precursors for the Colloidal Synthesis of Lead-Based Halide Perovskite Nanocrystals. J. Am. Chem. Soc. 2018, 140 (7), 2656-2664.

39. Weidman, M. C.; Seitz, M.; Stranks, S. D.; Tisdale, W. A., Highly Tunable Colloidal Perovskite Nanoplatelets through Variable Cation, Metal, and Halide Composition. ACS Nano 2016, 10 (8), 7830-7839.

40. Song, J. Z.; Li, J. H.; Xu, L. M.; Li, J. H.; Zhang, F. J.; Han, B. N.; Shan, Q. S.; Zeng, H. B., RoomTemperature Triple-Ligand Surface Engineering Synergistically Boosts Ink Stability, Recombination Dynamics, and Charge Injection toward EQE-11.6\% Perovskite QLEDs. Adv. Mater. 2018, 30 (30), 1800764.

41. Levchuk, I.; Osvet, A.; Tang, X.; Brandl, M.; Perea, J. D. o.; Hoegl, F.; Matt, G. J.; Hock, R.; Batentschuk, M.; Brabec, C. J., Brightly Luminescent and Color-Tunable Formamidinium Lead Halide Perovskite FAPbX3 (X = Cl, Br, I) Colloidal Nanocrystals. Nano Lett. 2017, 17 (5), 2765-2770.

42. Zhang, D. D.; Eaton, S. W.; Yu, Y.; Dou, L. T.; Yang, P. D., Solution-Phase Synthesis of Cesium Lead Halide Perovskite Nanowires. J. Am. Chem. Soc. 2015, 137 (29), 9230-9233.

43. Bodnarchuk, M. I.; Boehme, S. C.; ten Brinck, S.; Bernasconi, C.; Shynkarenko, Y.; Krieg, F.; Widmer, R.; Aeschlimann, B.; Gunther, D.; Kovalenko, M. V.; Infante, I., Rationalizing and Controlling the Surface Structure and Electronic Passivation of Cesium Lead Halide Nanocrystals. ACS Energy Lett. 2019, 4 (1), 63-74.

44. Maceiczyk, R. M.; Dumbgen, K.; Lignos, I.; Protesescu, L.; Kovalenko, M. V.; deMello, A. J., Microfluidic Reactors Provide Preparative and Mechanistic Insights into the Synthesis of Formamidinium Lead Halide Perovskite Nanocrystals. Chem. Mater. 2017, 29 (19), 8433-8439.

45. Lignos, I.; Protesescu, L.; Emiroglu, D. B.; Maceiczyk, R.; Schneider, S.; Kovalenko, M. V.; deMello, A. J., Unveiling the Shape Evolution and Halide-Ion-Segregation in Blue-Emitting Formamidinium Lead Halide Perovskite Nanocrystals Using an Automated Microfluidic Platform. Nano Lett. 2018, 18 (2), 1246-1252. 
46. Lignos, I.; Stavrakis, S.; Nedelcu, G.; Protesescu, L.; Demello, A. J.; Kovalenko, M. V., Synthesis of Cesium Lead Halide Perovskite Nanocrystals in a Droplet-Based Microfluidic Platform: Fast Parametric Space Mapping. Nano Lett. 2016, 16 (3), 1869-1877.

47. Udayabhaskararao, T.; Kazes, M.; Houben, L.; Lin, H.; Oron, D., Nucleation, Growth, and Structural Transformations of Perovskite Nanocrystals. Chem. Mater. 2017, 29 (3), 1302-1308.

48. De Roo, J.; Ibanez, M.; Geiregat, P.; Nedelcu, G.; Walravens, W.; Maes, J.; Martins, J. C.; Van Driessche, I.; Koyalenko, M. V.; Hens, Z., Highly Dynamic Ligand Binding and Light Absorption Coefficient of Cesium Lead Bromide Perovskite Nanocrystals. ACS Nano 2016, 10 (2), 2071-2081.

49. Guo, Y.; Shoyama, K.; Sato, W.; Nakamura, E., Polymer Stabilization of Lead(II) Perovskite Cubic Nanocrystals for Semitransparent Solar Cells. Adv. Energy Mater. 2016, 6 (6), 1502317.

50. Huang, H.; Chen, B.; Wang, Z.; Hung, T. F.; Susha, A. S.; Zhong, H.; Rogach, A. L., Water resistant CsPbX3 nanocrystals coated with polyhedral oligomeric silsesquioxane and their use as solid state luminophores in allperovskite white light-emitting devices. Chem. Sci. 2016, 7 (9), 5699-5703.

51. Kim, Y.; Yassitepe, E.; Voznyy, O.; Comin, R.; Walters, G.; Gong, X.; Kanjanaboos, P.; Nogueira, A. F.; Sargent, E. H., Efficient Luminescence from Perovskite Quantum Dot Solids. ACS Appl. Mater. Interfaces 2015, 7 (45), 25007-25013.

52. Pan, J.; Sarmah, S. P.; Murali, B.; Dursun, I.; Peng, W.; Parida, M. R.; Liu, J.; Sinatra, L.; Alyami, N.; Zhao, C.; Alarousu, E.; Ng, T. K.; Ooi, B. S.; Bakr, O. M.; Mohammed, O. F., Air-Stable Surface-Passivated Perovskite Quantum Dots for Ultra-Robust, Single- and Two-Photon-Induced Amplified Spontaneous Emission. J. Phys. Chem. Lett. 2015, 6 (24), 5027-5033.

53. Grisorio, R.; Di Clemente, M. E.; Fanizza, E.; Allegretta, I.; Altamura, D.; Striccoli, M.; Terzano, R.; Giannini, C.; Irimia-Vladu, M.; Suranna, G. P., Exploring the surface chemistry of cesium lead halide perovskite nanocrystals. Nanoscale 2019, 11 (3), 986-999.

54. Makarov, N. S.; Guo, S.; Isaienko, O.; Liu, W.; Robel, I.; Klimov, V. I., Spectral and Dynamical Properties of Single Excitons, Biexcitons, and Trions in Cesium-Lead-Halide Perovskite Quantum Dots. Nano Lett. 2016, 16 (4), 2349-2362.

55. Park, Y.-S.; Guo, S.; Makarov, N. S.; Klimov, V. I., Room Temperature Single-Photon Emission from Individual Perovskite Quantum Dots. ACS Nano 2015, 9 (10), 10386-10393.

56. Raino, G.; Nedelcu, G.; Protesescu, L.; Bodnarchuk, M. I.; Kovalenko, M. V.; Mahrt, R. F.; Stoeferle, T., Single Cesium Lead Halide Perovskite Nanocrystals at Low Temperature: Fast Single Photon Emission, Reduced Blinking, and Exciton Fine Structure. ACS Nano 2016, 10 (2), 2485-2490.

57. Tian, Y.; Merdasa, A.; Peter, M.; Abdellah, M.; Zheng, K.; Ponseca, C. S., Jr.; Pullerits, T.; Yartsev, A.; Sundstrom, V.; Scheblykin, I. G., Giant Photoluminescence Blinking of Perovskite Nanocrystals Reveals Single-Trap Control of Luminescence. Nano Lett. 2015, 15 (3), 1603-1608.

58. Utzat, H.; Shulenberger, K. E.; Achorn, O. B.; Nasilowski, M.; Sinclair, T. S.; Bawendi, M. G., Probing Linewidths and Biexciton Quantum Yields of Single Cesium Lead Halide Nanocrystals in Solution. Nano Lett. 2017, 17 (11), 6838-6846.

59. $\quad$ Becker, M. A.; Vaxenburg, R.; Nedelcu, G.; Sercel, P. C.; Shabaev, A.; Mehl, M. J.; Michopoulos, J. G.; Lambrakos, S. G.; Bernstein, N.; Lyons, J. L.; Stoferle, T.; Mahrt, R. F.; Kovalenko, M. V.; Norris, D. J.; Raino, G.; Efros, A. L., Bright triplet excitons in caesium lead halide perovskites. Nature 2018, 553 (7687), 189-193

60. Utzat, H.; Sun, W. W.; Kaplan, A. E. K.; Krieg, F.; Ginterseder, M.; Spokoyny, B.; Klein, N. D.; Shulenberger, K. E.; Perkinson, C. F.; Kovalenko, M. V.; Bawendi, M. G., Coherent single-photon emission from colloidal lead halide perovskite quantum dots. Science 2019, 363 (6431), 1068-1072.

61. Zhou, Y.; Zhao, Y., Chemical stability and instability of inorganic halide perovskites. Energy Environ. Sci. 2019, $12(5), 1495-1511$.

62. Zhou, Q.; Bai, Z.; Lu, W. G.; Wang, Y.; Zou, B.; Zhong, H., In Situ Fabrication of Halide Perovskite Nanocrystal-Embedded Polymer Composite Films with Enhanced Photoluminescence for Display Backlights. Adv. Mater. 2016, 28 (41), 9163-9168.

63. Lin, K.; Xing, J.; Quan, L. N.; de Arquer, F. P. G.; Gong, X.; Lu, J.; Xie, L.; Zhao, W.; Zhang, D.; Yan, C.; Li, W.; Liu, X.; Lu, Y.; Kirman, J.; Sargent, E. H.; Xiong, Q.; Wei, Z., Perovskite light-emitting diodes with external quantum efficiency exceeding 20 per cent. Nature 2018, 562 (7726), 245-248.

64. Lin, X.; Dai, X. L.; Pu, C. D.; Deng, Y. Z.; Niu, Y.; Tong, L. M.; Fang, W.; Jin, Y. Z.; Peng, X. G., Electrically-driven single-photon sources based on colloidal quantum dots with near-optimal antibunching at room temperature. Nat. Commun. 2017, 8.

65. Aharonovich, I.; Englund, D.; Toth, M., Solid-state single-photon emitters. Nat. Photonics 2016, 10 (10), 631-641.

66. Sercel, P. C.; Lyons, J. L.; Wickramaratne, D.; Vaxenburg, R.; Bernstein, N.; Efros, A. L., Exciton Fine Structure in Perovskite Nanocrystals. Nano Lett. 2019, 19 (6), 4068-4077.

67. Maceiczyk, R. M.; Lignos, I. G.; deMello, A. J., Online detection and automation methods in microfluidic nanomaterial synthesis. Curr. Opin. Chem. Eng. 2015, 8, 29-35. 
68. Lignos, I.; Maceiczyk, R.; deMello, A. J., Microfluidic Technology: Uncovering the Mechanisms of Nanocrystal Nucleation and Growth. Acc. Chem. Res. 2017, 50 (5), 1248-1257.

69. Phillips, T. W.; Lignos, I. G.; Maceiczyk, R. M.; deMello, A. J.; deMello, J. C., Nanocrystal synthesis in microfluidic reactors: where next? Lab Chip 2014, 14 (17), 3172-3180.

70. Locardi, F.; Cirignano, M.; Baranov, D.; Dang, Z.; Prato, M.; Drago, F.; Ferretti, M.; Pinchetti, V.; Fanciulli, M.; Brovelli, S.; De Trizio, L.; Manna, L., Colloidal Synthesis of Double Perovskite Cs2AgInCl6 and Mn-Doped Cs2AgInCl6 Nanocrystals. J. Am. Chem. Soc. 2018, 140 (40), 12989-12995.

71. Locardi, F.; Sartori, E.; Buha, J.; Zito, J.; Prato, M.; Pinchetti, V.; Zaffalon, M. L.; Ferretti, M.; Brovelli, S.; Infante, I.; De Trizio, L.; Manna, L., Emissive Bi-Doped Double Perovskite Cs2Ag1-xNaxInCl6 Nanocrystals. ACS Energy Lett. 2019, 1976-1982.

72. Yang, B.; Mao, X.; Hong, F.; Meng, W.; Tang, Y.; Xia, X.; Yang, S.; Deng, W.; Han, K., Lead-Free Direct Band Gap Double-Perovskite Nanocrystals with Bright Dual-Color Emission. J. Am. Chem. Soc. 2018, 140 (49), 17001-17006.

73. Yang, B.; Chen, J.; Yang, S.; Hong, F.; Sun, L.; Han, P.; Pullerits, T.; Deng, W.; Han, K., Lead-Free SilverBismuth Halide Double Perovskite Nanocrystals. Angew. Chem. Int. Ed. 2018, 57 (19), 5359-5363.

74. Abecassis, B.; Bouet, C.; Garnero, C.; Constantin, D.; Lequeux, N.; Ithurria, S.; Dubertret, B.; Pauw, B. R.; Pontoni, D., Real-Time in Situ Probing of High-Temperature Quantum Dots Solution Synthesis. Nano Lett. 2015, 15 (4), 2620-2626.

75. Abolhasani, M.; Coley, C. W.; Xie, L. S.; Chen, O.; Bawendi, M. G.; Jensen, K. F., Oscillatory Microprocessor for Growth and in Situ Characterization of Semiconductor Nanocrystals. Chem. Mater. 2015, 27 (17), 6131-6138.

76. Lignos, I.; Protesescu, L.; Stavrakis, S.; Piveteau, L.; Speirs, M. J.; Loi, M. A.; Kovalenko, M. V.; deMello, A. J., Facile Droplet-based Microfluidic Synthesis of Monodisperse IV-VI Semiconductor Nanocrystals with Coupled In-Line NIR Fluorescence Detection. Chem. Mater. 2014, 26 (9), 2975-2982.

77. Maceiczyk, R. M.; Bezinge, L.; deMello, A. J., Kinetics of nanocrystal synthesis in a microfluidic reactor: theory and experiment. React. Chem. Eng. 2016, 1, 261-271.

78. Nightingale, A. M.; Krishnadasan, S. H.; Berhanu, D.; Niu, X.; Drury, C.; McIntyre, R.; Valsami-Jones, E.; deMello, J. C., A stable droplet reactor for high temperature nanocrystal synthesis. Lab Chip 2011, 11 (7), $1221-1227$. 79. Schiener, A.; Magerl, A.; Krach, A.; Seifert, S.; Steinruck, H. G.; Zagorac, J.; Zahn, D.; Weihrich, R., In situ investigation of two-step nucleation and growth of CdS nanoparticles from solution. Nanoscale 2015, 7 (26), 1132811333.

80. Xie, L.; Shen, Y.; Franke, D.; Sebastian, V.; Bawendi, M. G.; Jensen, K. F., Characterization of Indium Phosphide Quantum Dot Growth Intermediates Using MALDI-TOF Mass Spectrometry. J. Am. Chem. Soc. 2016, 138 (41), 13469-13472.

81. Abdel-Latif, K.; Epps, R. W.; Kerr, C. B.; Papa, C. M.; Castellano, F. N.; Abolhasani, M., Facile RoomTemperature Anion Exchange Reactions of Inorganic Perovskite Quantum Dots Enabled by a Modular Microfluidic Platform. Adv. Funct. Mater. 2019, 29 (23), 1900712.

82. Baek, J.; Shen, Y.; Lignos, I.; Bawendi, M. G.; Jensen, K. F., Multistage Microfluidic Platform for the Continuous Synthesis of III-V Core/Shell Quantum Dots. Angew. Chem. Int. Ed. 2018, 57 (34), 10915-10918.

83. Bezinge, L.; Maceiczyk, R. M.; Lignos, I.; Kovalenko, M. V.; Demello, A. J., Pick a Color MARIA: Adaptive Sampling Enables the Rapid Identification of Complex Perovskite Nanocrystal Compositions with Defined Emission Characteristics. ACS Appl. Mater. Interfaces 2018, 10 (22), 18869-18878.

84. Epps, R. W.; Felton, K. C.; Coley, C. W.; Abolhasani, M., Automated microfluidic platform for systematic studies of colloidal perovskite nanocrystals: towards continuous nano-manufacturing. Lab Chip 2017, 17 (23), 4040 4047 .

85. Guidelli, E. J.; Lignos, I.; Yoo, J. J.; Lusardi, M.; Bawendi, M. G.; Baffa, O.; Jensen, K. F., Mechanistic Insights and Controlled Synthesis of Radioluminescent ZnSe Quantum Dots Using a Microfluidic Reactor. Chem. Mater. 2018, 30 (23), 8562-8570.

86. Li, C.; Ding, B.; Zhang, L.; Song, K.; Tao, S., 3D-printed continuous flow reactor for high yield synthesis of CH3NH3PbX3 (X = Br, I) nanocrystals. J. Mater. Chem. C 2019, 7, 9167-9174.

87. Lignos, I.; Stavrakis, S.; Kilaj, A.; deMello, A. J., Millisecond-Timescale Monitoring of PbS Nanoparticle Nucleation and Growth Using Droplet-Based Microfluidics. Small 2015, 11 (32), 4009-4017.

88. Polte, J.; Erler, R.; Thünemann, A. F.; Sokolov, S.; Ahner, T. T.; Rademann, K.; Emmerling, F.; Kraehnert, R., Nucleation and growth of gold nanoparticles studied via in situ small angle X-ray scattering at millisecond time resolution. ACS Nano 2010, 4 (2), 1076-1082.

89. Kuyper, C. L.; Budzinski, K. L.; Lorenz, R. M.; Chiu, D. T., Real-time sizing of nanoparticles in microfluidic channels using confocal correlation spectroscopy. J. Am. Chem. Soc. 2006, 128 (3), 730-731.

90. Qin, D.; Xia, Y.; Whitesides, G. M., Soft lithography for micro- and nanoscale patterning. Nat. Protoc. 2010, $5(3), 491-502$. 
91. Chirvony, V. S.; González-Carrero, S.; Suárez, I.; Galian, R. E.; Sessolo, M.; Bolink, H. J.; Martínez-Pastor, J. P.; Pérez-Prieto, J., Delayed Luminescence in Lead Halide Perovskite Nanocrystals. J. Phys. Chem. C 2017, 121 (24), 13381-13390.

92. Rabouw, F. T.; Kamp, M.; van Dijk-Moes, R. J. A.; Gamelin, D. R.; Koenderink, A. F.; Meijerink, A.; Vanmaekelbergh, D., Delayed Exciton Emission and Its Relation to Blinking in CdSe Quantum Dots. Nano Lett. 2015, 15 (11), 7718-7725.

93. Rabouw, F. T.; van der Bok, J. C.; Spinicelli, P.; Mahler, B.; Nasilowski, M.; Pedetti, S.; Dubertret, B.; Vanmaekelbergh, D., Temporary Charge Carrier Separation Dominates the Photoluminescence Decay Dynamics of Colloidal CdSe Nanoplatelets. Nano Lett. 2016, 16 (3), 2047-2053.

94. Pope, M.; Swenberg, C. E.; Pope, M., Electronic processes in organic crystals and polymers. 2nd ed.; Oxford University Press: New York, 1999. 